\title{
Novel Properties of Semiconductor Nanowires
}

\author{
Kruti Wohraa, Arun Kumar Diwakara*, Anant G. Kulkarni ${ }^{\mathrm{b}}$
}

aDepartment of Physics, Kalinga University, Raipur, 492101, Chhattisgarh, India

bSiddhivinayak Technical Campus, Khamgaon Road, Shegaon, 444203, Maharashtra, India

\section{Article Info}

Volume 8, Issue 5

Page Number : 345-354

\section{Publication Issue}

September-October-2021

\section{Article History}

Accepted : 05 Oct 2021

Published : 14 Oct 2021

\section{ABSTRACT}

Semiconductor nanowires guarantee to give the structure squares to another age of nanoscale electronic and optoelectronic gadgets and display novel electronic and optical properties inferable from their special underlying onedimensionality and conceivable quantum confinement impacts in two measurements. With an expansive choice of creations and band structures, these one-dimensional semiconductor nanostructures are viewed as the basic segments in a wide scope of potential nanoscale device applications. This review paper explains the basic properties showed by semiconductor nanowires. Novel properties including nanowire miniature hole lasing, phonon transport, interfacial security, and synthetic detecting are reviewed.

Keywords: Semiconductor, Nanowires and Properties of Nanowires.

\section{INTRODUCTION}

Semiconductor nanowires have shown energizing properties for nanophotonic, sensors, energy innovations, and end-of-guide and past guide electronic device. Semiconductor nanowires (NWs) are nanoscale materials that have been imagined to have applications in cutting-edge gadgets to upgrade usefulness, act superiorly, and take into consideration high integrability and decreases in cost. Lately, an examination into semiconductor NWs has quickly evolved and has been broadly researched in a wide scope of photograph energy applications, including photograph identifiers, photograph catalysis, photograph voltaic, and thermoelectric.

\section{PROPERTIES}

The advancement capability of nanowires has in this way touched off an interdisciplinary exertion planning to the showing of novel and proficient strategies for the acknowledgment of these nanoscale structures, their control, the investigation of their compound, underlying, optical, and electrical properties, and their execution in unique device models. Having this at the top of the priority list, the current review article intends to give the peruser a basic overview of the property of semiconductor nanowires.

The best candidate for nanowire is the carbon nanotubes, nanocrystals, and semiconductor 
nanowires. Semiconductor nanowires have plenty of features that are critical for destiny applications. They may be fabricated with uniform composition up to a diameter of $3 \mathrm{~nm}$ [1]. Furthermore, a p or n-type doping necessary for maximum cloth applications has been demonstrated in many instances. Once doping is completed, the next step would be to try and make subject-effect transistors. Consequently, the device length has been shrinking up to the nanoscale length. Issues arise at this size because that is the atomic length range and physics starts to trade. Many properties had been observed to alternate on the nanoscale variety. in particular, it has been determined that the bandgap of semiconducting nanowires decreases with diameter because of quantum confinement. The fine version for the phenomenon is a $1 / d^{n}$ with $\mathrm{d}$ being the diameter and $1<n<2$. For silicon nanowires, $\mathrm{n}$ become observed to be 1.7 even as for InP, it became located to be 1 forty-five [2]. One of the most known assets is the hall-Petch effect feature of polycrystalline solids. This asset suggests that the yield power and hardness of micro structured polycrystalline will increase with decreasing length. It changed into eventually proven that the hall-Petch impact breaks down on the nanoscale range in which the substances soften as a substitute [3]. Different similar properties have been found and others can be on the manner. We might simply mention also the melting temperature that is regarded to decrease for nanowires. Many other residences can be referred to mainly in the regions of lasing, phonon delivery, magnetic effects, and lots greater. Semiconductor nanowires provide many ability packages in electronics and optoelectronic gadgets [4-5]. The fundamental challenge for physicists is to quantify such residences and discover materials with unique properties well worth the use of in enterprise because experiments are expensive, the fine technique is to use laptop simulations which might be acknowledged to yield properties corresponding to experiments. That is what led me to this study and sustained my motivation during these years. indeed, the bandgap increases with the lower nanowire diameter due to the quantum confinement impact, which allows the control of photoluminescence results over a huge variety of wavelengths [6-7]. Properties of semiconductor nanowire are shown in the figure:

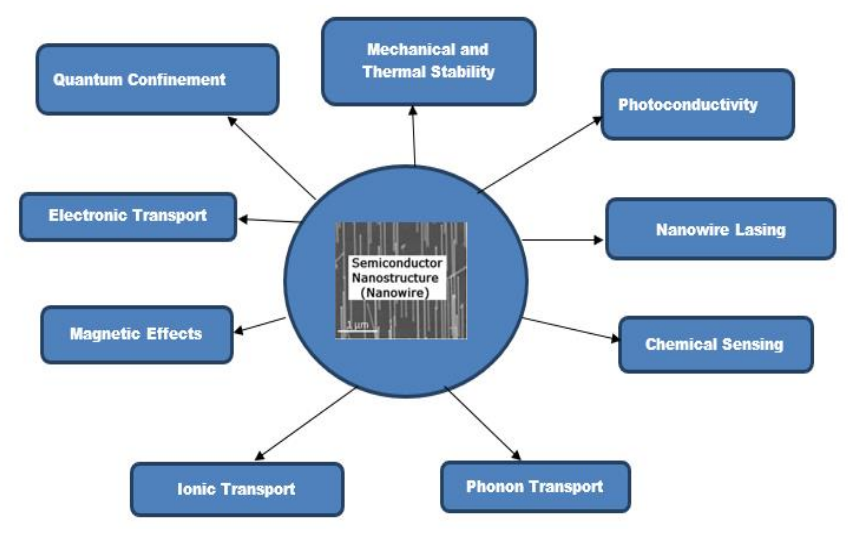

Figure 1: Properties of Semiconductor Nanowire.

\section{Quantum Confinement}

Quantum confinement is nothing but a change of optical and electronic properties when the substance sampled is of ample small length - typically 10 nanometers $(\mathrm{nm})$ or much less. The size of the nanostructure is inversely proportional to the bandgap that is bandgap increases as size decreases. It's been observed that quantum confinement owes its starting place to surface stress attributable to floor passivation and surface reconstruction and therefore thin nanowires may be amorphous-shell (a-Si) / crystalline-center (c-Si) nanostructure (nanowires). A new definite investigation of the impact of dimensionality on restriction in indium phosphide (InP) wires reasoned that the size reliance of the bandgap in wires is more fragile than in spots by the sum anticipated from straightforward hypothesis [8]. In any case, the total bandgap moves in InP wires $\left(\Delta E_{g}=1 / d^{1.45}\right)$ didn't follow the molecule in-a-case expectation (i.e., $1 / d^{2}$ ), showing that exact medicines of constraint require higher-request estimations to represent band structure. 
Bandgap tunability and the subsequent changes in discharge/assimilation energies have been widely explored in nanoparticles and inhomogeneous nanorods made of materials with sensibly huge exciton Bohr radii, like CdSe and InAs [9-11]. Single InP nanowires have likewise gotten consideration [12]. The photoluminescence (PL) of very much scattered, size-chose InP wires were found to blue shift with diminishing size for widths under $20 \mathrm{~nm}$. Light retention and discharge in a nanowire are exceptionally polarization ward with the end goal that the PL power is a maximum for polarization corresponding to the long hub of the nanowires [1314]. This polarization anisotropy is conceivable because of the sharp dielectric contrast between nanowires and their environmental factors, which can be misused to make different devices. Further developing the vehicle network by supplanting nanorods with Cadmium telluride (CdTe) tetrapod branched nanocrystals should upgrade future cell execution [15]. Moreover, the cover of the CdTe ingestion profile with the sunlight-based range is tunable by $0.5 \mathrm{eV}$ by changing the breadth of the four arms of the tetrapod's, along these lines empowering the band designing of a sun-oriented cell.

The zinc oxide $(\mathrm{ZnO})$ / zinc magnesium oxide (ZnMgO) multiple quanta well (MQW) structures nanostructure concentrate by Park and Pennycook (2003) which is a fine illustration of flexible quantum devices dependent on individual heterostructures [16]. They utilized metalorganic chemical vapor deposition (MOCVD) or Metalorganic vapor-phase epitaxy (MOVPE)to develop nanostructures containing a grouping of dainty $\mathrm{ZnO}$ wells isolated by epitaxial $Z n_{0.8} M g_{0.2} O$ layers.

In addition to true multiple quanta well 1-D device, conned-core-sheath structure's nanowire heterostructures provide an extraordinary geometry for uses in optical-to-electrical or electrical-to-optical (optoelectronics). We as of late exhibited UV lasing from optically pumped $A l_{x} G a_{1-x} N / G a N$ center sheath structure quantum nanowires [17]. Typically, Gallium nitride $(\mathrm{GaN})$ nanowires with widths under $100 \mathrm{~nm}$ are too cracked to even think about supporting laser depression modes. Encompassing slender $\mathrm{GaN}$ wires with a material of bigger bandgap and more modest refractive index makes a design with photon confinement and synchronous exciton(waveguiding).

\section{Ionic Transport}

There is various type of transport mechanism like Space charge limited current, hopping transport and thermally activated transport in nanostructures. Semiconductor nanostructures represent a special system with three, two, or one quantum-confined direction for the transportation of electrons [18]. The nanowires carrier transport can be quite specific than in the bulk, in particular for semiconductor nanowires with small diameters like $<10 \mathrm{~nm}$, wherein the interaction of carrier-surface becomes the maximum important source of scattering. Quantum confinement outcomes may also come to be critical, with an effect on the band structure affecting additionally the mobility of carrier [19]. In the year 2004, Hahm and Lieber investigated that semiconductor nanowire field-effect devices work as sensors for the detection of single-stranded deoxyribonucleic acid (DNA) [20].

The ionic vehicle through nanoscale channels is getting expanding consideration inferable from late tests that report tweak of particle flows during the entry of single atoms of DNA or protein through the protein particle channel $\alpha$-hemolysin [21]. The chance of quick DNA sequencing by observing the ionic conductance marks of passing nucleotide oligomers has provoked the combination of counterfeit nanopores and the investigation of biomolecular transport through them [22]. Nanostructure gives a one-of-a-kind high-perspective proportion divert in which to examine particle transport and a liquid stream. Hypothetical treatment 
of particle conduct in gated silica nanotubes proposes that when the cylinder breadth is more modest than the Debye length, and applied entryway inclination can remove particles of like charge and produce a unipolar arrangement of counter-particles inside the channel [23]. Additionally, the 5-20 $\mu \mathrm{m}$ length of the nanotubes divert now being manufactured in this research facility opens up the chance of imaging and controlling single atoms as they go through a cylinder.

\section{Mechanical and Thermal Stability}

The high surface-to-volume ratios and small sizes of one-dimensional nanostructures provide them with the heterogeneity of useful and compelling mechanical properties. In present years, nanowires have been investigated extensively because they have wide use in different regions, along with nanomechanical and nanoelectronics devices. One of the mechanical properties of the nanowire is critically decreased when the temperature will increase from $20 \mathrm{k}$ to $180 \mathrm{k}$, however, grow to be much less intense at high temperature. Atomistic displaying completed by Schiotz focuses on a change from separation intervened respecting grain limit sliding at little crystallite sizes as the essential clarification for the atypical greatest in the strength of metallic polycrystalline solids [24]. They report an unconstrained face-centered cubic (FCC) to bodyfocused tetragonal stage change in nanowires with a 100 starting precious stone direction and crosssectional region under $4 \mathrm{~nm}^{2}$. The progress is nucleated at the finishes of the nanowire and propagates internally at a $10^{\text {th }}$ the speed of sound in gold. No such impact was found in wires with 110 or 111 development headings, as these directions highlight surfaces that need adequate pressure to beat mass dependability. It is realized that the dissolving temperature of a precious stone is contrarily relative to its compelling range for grains less than 20 to 40 $\mathrm{nm}$ [25-27].
Past literature has shown that the electric conductivity of semiconductor nanowire (GeNW) can be enhanced under high tensile strain [28]. In the year 2016; Po-Yu Yang and team members constructed four special sizes of Germanium nanowire (GeNW) were built and their thermal stabilities had been analyzed. The first three types of single-shell Germanium nanowires (GeNW) are name hexagon, pentagon, and helix GeNW, with radii of 2.4 Angstrom (A), 2.0 A, and 1.8 Angstrom respectively. There's additionally one center-shell GeNW named 7-1 GeNW which demonstrates $7 \mathrm{Ge}$ atoms in its outer shell, $1 \mathrm{Ge}$ atom within the middle, and a radius of $2.9^{\circ}$ A. the size and temperature dependence of the tensile behavior and mechanical property are investigated to technique an actual environment [29].

Transmission Electron Microscopy (TEM) showed that the closures of sheathed wires started to liquefy $280{ }^{\circ} \mathrm{C}$ underneath the mass dissolving temperature, with the fluid fronts compromising $80^{\circ} \mathrm{C}$ short of the mass worth. The last examination is a nanowire illustration of slender dissolving (the Gibbs-Thomson impact), in which the strong fluid harmony of material is moved to bring down temperature by control in a sheath having great wetting properties with the fluid [30].

Nanowire blend methods can yield single-glasslike structures with a much lower thickness of line abandons than is normally found in mass materials. As a result, one-dimensional nanostructures frequently include mechanical strength, firmness, and sturdiness moving toward the hypothetical furthest reaches of amazing gems, making them appealing for use in composites and as actuators in nanoelectromechanical frameworks (NEMS). In 1997, the Lieber bunch spearheaded the utilization of nuclear power microscopy (AFM) to decide the mechanical properties of individual $\mathrm{SiC}$ nanostructure nanorods that were stuck toward one side to a strong substrate [31]. 
Notwithstanding mechanical portrayal, a few examinations have exhibited mechanical activation dependent on the extraordinary highlights of onedimensional designs. For example, adaptable Silicon dioxide nanoparticles ( $\mathrm{SiO} 2 \mathrm{NPs}$ ) helices warmed by an electron bar show extension withdrawal conduct like that of spring [32]. A new report of a $5.5 \mathrm{fg}$ mass location utilizing bigger silicon ( $\mathrm{Si}$ ) cantilevers in surrounding conditions proclaims the wide utilization of nanowires in power microscopy, high-recurrence hardware, and calorimetric in the quantum system [33].

\section{Nanowire Lasing}

Semiconductor nanowire lasers are nano-scaled lasers that can be embedded on chips and constitute and strengthen for information processing and computing applications. Nanowire lasers are coherent mild assets as every other laser device, with the gain of operating on the nanoscale. Nanowires with level-end aspects can be abused as optical reverberation holes to create cognizant light on the nanoscale. Room temperature UV lasing has been shown in our research facilities for the Gallium nitride ( $\mathrm{GaN})$ and Zinc oxide $(\mathrm{ZnO})$ nanowires frameworks with brushes, epitaxial exhibits, and single nanowires [34]. $\mathrm{ZnO}$ and $\mathrm{GaN}$ are wide bandgap semiconductors (3.37, $3.42 \mathrm{eV}$ ) appropriate for UV-blue optoelectronic applications.

The most helpful applications for nanowire lasers necessitate that they are coordinated in circuits and enacted by electron-infusion as opposed to optical siphoning. Lieber and associates have gained ground toward this path by collecting n-type Cadmium sulfide (CdS) nanowire Fabry-Perot holes on $\mathrm{p}-\mathrm{Si}$ wafers to shape the necessary heterojunction for electrically determined lasing [35]. More vigorous get-together techniques suitable to a bigger assortment of materials will empower the utilization of infusion nanolasers in detecting, optical correspondences, and test microscopy.

\section{Phonon Transport}

Phonon transport is relied upon to be incredibly hindered in flimsy (i.e., $\mathrm{d}<\Lambda$, where $\mathrm{d}$ is the width and $\Lambda$ is the phonon mean freeway) one-dimensional nanostructures because of expanded limit dissipating and decreased phonon bunch speeds coming from phonon control. Definite models of phonon heat conduction in tube-shaped and rectangular semiconducting nanowires that consider adjusted scattering relations and extremely significant dispersing measures anticipate an enormous decline (>90\%) in the cross-section warm conductivity of wires many nanometers in width [36-37].

Size-subordinate warm conductivity in nanostructures presents a major obstacle in the drive toward scaling down in the semiconductor. Ten years prior, the Dresselhaus bunch anticipated that dimensionless figure of merit (ZT) can be expanded above mass qualities in slim nanowires via cautiously fitting their breadths, organizations, and transporter fixations. These remaining parts are to be tentatively affirmed [38].

A few exploration bunches are currently manufacturing nanowires for thermoelectric (TE) applications. Varieties of Bismuth telluride (Bi2Te3) and Bismuth antimonide (BiSb) wires filled electrochemically in anodic alumina layouts and afterward embedded in a thermally protecting network may before long give valuable TE materials [39-40]. Future seebeck and electronic investigations of further developed superlattice nanowires are expected to direct the designing of these materials for TE applications.

Phonon transport in mesoscopic one-dimensional frameworks was taken close to its ultimate limit with the estimation by Roukes and associates of the widespread quantum of warm conductance, Gth $\pi 2 \mathrm{k}$ 2T/3h [41]. They have since proceeded to look at the subtleties of phonon dissipating in lithographically 
pre-arranged nanobeams of electrically leading and protecting materials [42].

\section{Photoconductivity and Chemical Sensing}

Electronic conductivity in semiconductor nanowires, belts, and cylinders is substantially upgraded by presenting these designs to photons of energy more noteworthy than their band gaps. We illustrated that photoconductivity in ZnO NANOWIREs could be misused to make quick and reversible UV optical switches with ON-OFF changing proportions of four to six significant degrees under low-power $365 \mathrm{~nm}$ light [43]. Since the size and rot season of the photo response is an exceptionally deep gouge on the presence of encompassing $\mathrm{O}_{2}$, we recommended that the photocurrent in n-type oxide nanowires is a result of electron-opening pair development and electron doping brought about by the photograph prompted desorption of oxidizing surface species, including oxygen. We proceeded to use the touchy reliance of nanowire conductivity on adsorbing ate atoms to manufacture the main single-glasslike nanowire gas sensor [44]. A progression of studies by different gatherings later stretched out nanowire-based gas detecting to Indium Oxide (In2O3) nanowires [45], Zinc Oxide ( $\mathrm{ZnO})$ belts [46], Titanium dioxide $\left(\mathrm{TiO}_{2}\right)$ tubes exhibits [47] and polycrystalline tin oxide $\left(\mathrm{SnO}_{2}\right)$ nanowires [48]. Nanowire compound sensors frequently work through substance gating prompted by the surface adsorption of analyte atoms, albeit other detecting instruments exist [49-50].

The extremely high surface-to-volume proportions of slim one-dimensional nanostructures enrich them with intrinsically high affectability and short reaction time; in any case, selectivity is a significant issue, particularly in the recognition of gases. Selectivity in nanowire sensors is all the more handily tended to in fluid media, where ligand-receptor restricting (e.g., biotin-streptavidin) and other surface functionalization plans can give atomic separation [51]. It is a decent wagered that nanowire and Carbon nanotubes (CNTs) based synthetic detecting will be among the primary significant business applications for one-dimensional nanostructures.

\section{Magnetic Effects}

The attractive properties of solids can show size reliance as a result of a few impacts, including the impact of surfaces, the beginning of transporter binding, and the decrease of design size beneath that of a solitary attractive area. It is feasible to improve or even actuate attractive conduct by changing the dimensionality of a framework. For instance, the wrecked evenness of a surface can create goliath attractive anisotropy energy in attractive adatoms [52] The investigation of electron transport in suspended chains of particles recommends that specific nonmagnetic frameworks, like Palladium (Pd) and platinum $(\mathrm{Pt})$ become attractive in such math and act as ferromagnetic captivated conduction channels (with the quantized vehicle at a large portion of a conductance quantum) [53]. Most examinations on attractive one-dimensional designs use cathode set ferromagnetic nanowires filled in the enormous region, variable-thickness exhibits in anodic alumina (AAO), or track-carve layouts [54].

AAO template ferromagnetic nanowires are demonstrating value in the arising field of biomagnetic in which attractive nanostructures give away to detect biomolecules, sort cells, and perform other organic controls. For example, taking note of that the huge remainder charge intrinsic to nanowires licenses their utilization in low-field conditions, the Reich bunch has exhibited the substance functionalization of $\mathrm{Au} / \mathrm{Ni}$ wires for biosensing and fostered a way to deal with attractively trap single wires in arrangement [55-56].

\section{Electronic Transport}

One striking wonder in the mesoscopic area is the quantization of electrical conductance, which happens when a semi one-dimensional electron gas is 
made to connect two-electron repositories, as in a semiconductor point contact, mechanical break intersection, or carbon nanotube [57-59]. Under ideal ballistic conditions, each twist degenerate quantum channel contributes a unit of $2 e^{2} / h$ to the electrical conductance. At the hour of this composition, everything except one of the implied instances of quantized conductance in nanowires depended on chains of metal iotas instead of detached, artificially orchestrated one-dimensional nanostructures.

The perception of quantum transport impacts in evident metal or semiconductor nanowires requires structures with widths practically identical to the Fermi frequency (regularly several $\mathrm{nm}$ for semiconductors, $<1 \mathrm{~nm}$ for metals). The trouble in combining such wires and furnishing them with contacts having high transmission coefficients somewhat clarifies the deficiency of research around here. In any case, a new report of solid Coulomb bar conduct in solid Indium phosphide ( $\mathrm{InP}$ ) nanowires at $0.35 \mathrm{~K}$ pleasantly outlines the plausibility of the exploration. This investigation supplements the means taken toward nanowire-based quantum devices by Samuelson and associates with their demonstration of a thunderous burrowing diode and a solitary electron semiconductor utilizing individual Molecular-beam epitaxy (MBE) developed heterostructure nanowires at temperatures under 12 $\mathrm{K}[60-62]$.

Compositionally adjusted (heterostructure) nanowires that highlight inside $\mathrm{p}-\mathrm{n}$ intersections have empowered the advancement of single nanowire LEDs and different devices. Ongoing models incorporate the vapor-liquid-solid method (VLS) developed $\mathrm{Si}$ and $\mathrm{GaN}$ nanowire rectifiers and $\mathrm{InP}$ nanowire LEDs, just as amending multi-facet metal bars manufactured by electrodeposition. At last, we note that different parts of charge transport in both single segment and balanced nanowires as of late got hypothetical consideration [63-66].

\section{CONCLUSION}

In this review; we have presented novel properties of semiconductor nanowires. Quantum confinement, Mechanical and Thermal Stability, nanowire Lasing, Electronic Transport, Magnetic Effects, Photoconductivity, and Chemical Sensing, Phonon Transport, nanowire Leasing are discussed.

\section{IV.REFERENCES}

[1] Y. Wu, Y. Cui, L. Huynh, C. J. Barrelet, C. Bell, and C. M. Lieber, (2004) Controlled Growth and Structures of Molecular-Scale Silicon Nanowires, Nano Letter 4, 433.

[2] H. Lu, J. Li, R. A. Loomis, L. M. Wang, and W. E Buhro, (2003) Two-versus three-dimensional quantum confinement in indium phosphide wires and dots, Nat. Mater. 2, 517.

[3] J. Schiotz and K. W. Jacobsen, (2003) A maximum in the strength of nanocrystalline copper, Science, 301, 1357.

[4] H. Ch. Weissker, J. Furthmuller, and F. Bechstedt, (2002) Structure- and spindependent excitation energies and lifetimes of $\mathrm{Si}$ and $\mathrm{Ge}$ nanocrystals from ab initio calculations, Phys. Rev. B, 65, 155327.

[5] D. Erts, B. Polyakov, B. Daly, M. A. Morris, S. Ellingboes, J. Boland, J. D Holmes, (2006) High-Density Arrays of Germanium Nanowire Photoresistors, J. Phys. Chem. B, 110, 820.

[6] L. Pizzagalli, G. Giulia, J. E. Klepeis, and F. Gygi, (2001) Structure and stability of germanium nanoparticles, Phys. Rev. B 63, 165324.

[7] T. V. Torchynska, G. Polupan, J. P. Gomez and A.V. Kolobov, (2003) Microelectronics Journal, 34, 541.

[8] Yu H, Li J, Loomis RA, Wang L-W, Buhro WE. (2003) Two- versus three-dimensional quantum confinement in indium phosphide wires and dots. Nat. Mater, 2, 517- 20. 
[9] Murray CB, Kagan CR, Bawendi MG. (2000) Synthesis and characterization of monodisperse nanocrystals and close packed nanocrystal assemblies. Annu. Rev. Mater. Sci., 30, 545-610.

[10] Li L-S, Hu J, Yang W, Alivisatos PA. (2001) Band gap variation of size- and shapecontrolled colloidal CdSe quantum rods. Nano Lett., 1, 349-51.

[11] Kan S, Mokari T, Rothenberg E, Banin U. (2003) Synthesis and size-dependent properties of zinc-blende semiconductor quantum rods. Nat.Mater. 2, 155-58.

[12] Gudiksen MS, Wang J, Lieber CM. (2002) Sizedependent photoluminescence from single indium phosphide nanowires. J. Phys. Chem. B, 106, 4036-39.

[13] Wang J, Gudiksen MS, Duan X, Cui Y, Lieber CM. (2001) Highly polarized photoluminescence and photodetection from single indium phosphide nanowires. Science, 293, 1455-57.

[14] Qi J, Belcher AM, White JM. (2003) Spectroscopy of individual silicon nanowires. Appl. Phys. Lett. 82, 2616-18.

[15] Manna L, Milliron D J, Meisel A, Scher E C, Alivisatos A P. (2003) Controlled growth of tetrapod-branched inorganic nanocrystals. Nat. Mater 2, 382-85.

[16] Park, WI, Yi G-C, Kim M, Pennycook SJ. (2003) Quantum Confinement Observed inZnO/ZnMgO Nanorod heterostructures. Adv. Mater. 15, 526-29.

[17] Choi H-J, Johnson JC, He R, Lee S-K, Kim F, et al. (2003) Self-organized GaN quantum wire UV lasers. J. Phys. Chem. B, 107, 8721-25.

[18] M. A. Rafiq, (2018) Carrier transport mechanisms in semiconductor nanostructures and devices, Journal of Semiconductors, 39(6), 1-13.

[19] R. Rurali, (2010) Colloquium: Structural, electronic, and transport properties of $\mathrm{Si}$ nanowires, Reviews of Modern Physics, 82,
427.

[20] J. I. Hahm and C. M. Lieber, (2004) Direct ultrasensitive electrical detection of DNA and DNA sequence variations using nanowire nano sensors, Nano Letters, 4(1), 51.

[21] Meller A, Nivon L, Brandin E, Golovchenko J, Branton D. (2000) Rapid nanopore discrimination between single polynucleotide molecules. Proc. Natl. Acad. Sci. USA 97, 107984.

[22] Li J, Stein D, McMullan C, Branton D, Aziz MJ, Golovchenko J. (2001) Ion-beam sculpting at nanometer length scales. Nature 412, 166-69.

[23] Daiguji H, Yang P, Majumdar A. (2004) Ion transport in nanofluidic channels. Nano Lett. 4, 137-142

[24] Schiotz J, Jacobsen KW. (2003) A maximum in the strength of nanocrystalline copper. Science 301, 1357-59.

[25] Buffat P, Borel J-P. (1976) Size effect on the melting temperature of gold particles. Phys. Rev. A 13, 2287-98.

[26] Grulseren O, Ercolessi F, Tosatti E. (1995) Premelting of thin wires. Phys. Rev. B 51, 7377-80.

[27] Schmidt $M$, Kusche $R$, von Issendorff $B$, Haberland H. (1998) Irregular variations in the melting point of size-selected atomic clusters. Nature 393, 238-40.

[28] Y. Li, R. Clady, J. Park, S. V. Thombare, T. W. Schmidt, M. L. Brongersma and P. C. McIntyre, (2014) Ultrafast electron and phonon response of oriented and diameter-controlled germanium nanowire arrays, Nano Lett., 14, 3427-3431.

[29] Po-Yu Yang, Shin-Pon Ju, Zhu-Min Lai, JinYuan Hsieh, and Jennsen Lin, (2016) The mechanical properties and thermal stability of ultrathin germanium nanowires, RSC Advances, 105713-105722.

[30] Christenson HK. (2001) Confinement effects on freezing and melting. J. Phys. Condens. Matter, 13, 95-133.

[31] Wong WW, Sheehan PE, Lieber CM. (1997) 
Nanobeam mechanics: elasticity, strength, and toughness of nanorods and nanotubes. Science, 277, 1971-75.

[32] Zhang HF, Wang CM, Buck EC, Wang LS. (2003) Synthesis, characterization, and manipulation of helical $\mathrm{SiO}$ nano springs. Nano Lett. 3, 577-80.

[33] Lavrik NV, Datskos PG. (2003) Femtogram mass detection using photothermally actuated nanomechanical resonators. Appl. Phys. Lett. 82, 2697-99.

[34] Johnson JC, Yan H, Yang P, Saykally RJ. (2003) Optical cavity effects in $\mathrm{ZnO}$ nanowire lasers and waveguides. J. Phys. Chem. B 107, 8816-28.

[35] Duan X, Huang Y, Agarwai R, Lieber CM. (2003) Single-nanowire electrically driven lasers. Nature 421, 241-45.

[36] Zou J, Balandin A. (2001) Phonon heat conduction in a semiconductor nanowire. J. Appl. Phys. 89, 2932-38.

[37] Lu X, Chu JH, Shen WZ. (2003) Modification of the lattice thermal conductivity in semiconductor rectangular nanowires. J. Appl. Phys. 93, 1219-29.

[38] Hicks LD, Dresselhaus MS. (1993) Effect of quantum-well structures on the thermoelectric figure of merit. Phys. Rev. B 47, 12727-31.

[39] Martin-Gonzalez M, Snyder GJ, Prieto AL, Gronsky R, Sands T, Stacy AM. (2003) Direct electrodeposition of highly dense $50 \mathrm{~nm} \mathrm{Bi}$ 2Se3-yTe nanowire arrays. Nano Lett. 3, 97377.

[40] Prieto AL, Martin-Gonzalez M, Kenayi J, Gronsky R, Sands T, Stacy AM. (2003) The electrodeposition of high-density, ordered arrays of BiSb nanowires. J. Am. Chem. Soc. 125, 2388-89.

[41] Schwab K, Henriksen EA, Worlock JM, Roukes ML. (2000) Measurement of the quantum of thermal conductance. Nature 404, 974-77.

[42] Fon W, Schwab KC, Worlock JM, Roukes ML. (2002) Phonon scattering mechanisms in suspended nanostructures from 4 to $40 \mathrm{~K}$. Phys. Rev. B 66, 045302.

[43] Kind H, Yan H, Law M, Messer B, Yang P. (2002) Nanowire UV photodetectors and optical switches. Adv. Mater. 14, 158-60.

[44] Law M, Kind H, Kim F, Messer B, Yang P. (2002) NO photochemical sensing with $\mathrm{SnO}$ nanoribbons at room temperature. Angew. Chem. Int. Ed. 41, 2405-8.

[45] Li C, Zhang D, Liu X, Han S, Tang T, Han J, Zhou C. (2003) In O nanowires as chemical sensors. Appl. Phys. Lett. 82, 1613-15.

[46] Comini E, Faglia G, Sberveglieri G, Pan Z, Wang ZL. (2002) Stable and highly sensitive gas sensors based on semiconducting oxide nanobelts. Appl. Phys. Lett. 81, 1869-71.

[47] Varghese OK, Gong D, Paulose M, Ong KG, Dickey EC, Grimes CA. (2003) Extreme changes in the electrical resistance of titania nanotubes with hydrogen exposure. Adv. Mater. 15, 62427.

[48] Kolmakov A, Zhang Y, Cheng G, Moskovits M. (2003) Detection of CO and Ousting tin oxide nanowire sensors. Adv. Mater. 15, 997-1000.

[49] Favier F, Walter EC, Zach MP, Benter T, Penner RM. (2001) Hydrogen sensors and switches from electrodeposited palladium microwire arrays. Science 293, 2227-31.

[50] Li CZ, He X, Bogozi A, Bunch JS, Tao NJ. (2000) Molecular detection based on conductance quantization of nanowires. Appl. Phys. Lett. 76, 1333-35.

[51] Cui Y, Wei Q Park H, Lieber CM. (2001) Nanowire nano sensors for highly sensitive and selective detection of biological and chemical species. Science 293, 1289-92.

[52] Gambardella P, Rusponi S, Veronese M, Dhesi SS, Grazioli C, et al. 2003. Giant magnetic anisotropy of single cobalt atoms and nanoparticles. Science 300, 1130-33.

[53] Rodrigues V, Bettini J, Silva PC, Ugarte D. (2003) Evidence for spontaneous spin-polarized 
transport in magnetic nanowires. Phys. Rev. Lett. 91, 096801

[54] Sellmyer DJ, Zheng M, Skomski R. (2001) Magnetism of $\mathrm{Fe}$, Co and Ni nanowires in selfassembled arrays. J. Phys. Condens. Matter 13, R433-60.

[55] Reich DH, Tanase M, Hultgren A, Bauer LA, Chen CS, Meyer GJ. (2003) Biological applications of multifunctional magnetic nanowires. J. Appl. Phys. 93, 7275-80.

[56] Tanase M, Silevitch DM, Hultgren A, Bauer LA, Searson PC, et al. (2002) Magnetic trapping and self-assembly of multicomponent nanowires. J. Appl. Phys. 91, 8549-51.

[57] van Wees BJ, van Houten $H$, Beenakker CWJ, Williamson JG, Kouwenhoven LP, et al. (1988) Quantized conductance of point contacts in a two-dimensional electron gas. Phys. Rev. Lett. 60, 848-50.

[58] Muller CJ, van Ruitenbeek JM, de Jongh LJ. (1992) Conductance and supercurrent discontinuities in atomic-scale metallic constrictions of variable width. Phys. Rev. Lett. 69, 140-43.

[59] Frank S, Poncharal P, Wang ZL, de Heer WA. (1998) Carbon nanotube quantum resistors. Science 280, 1744-46.

[60] De Franceschi S, van Dam JA, Bakkers EPAM, Feiner LF, Gurevich L, Kouwenhoven LP. (2003) Single-electron tunneling in InP nanowires. Appl. Phys. Lett. 83, 344-46.

[61] Bjork MT, Ohlsson BJ, Thelander C, Persson AI, Deppert K, et al. (2002) Nanowire resonant tunneling diodes. Appl. Phys. Lett. 81, 4458-60.

[62] Thelander C, Martensson T, Bjork MT, Ohlsson BJ, Larsson MW, et al. (2003) Single-electron transistors in heterostructure nanowires. Appl. Phys. Lett. 83, 2052-54.

[63] Cheng G, Kolmakov A, Zhang Y, Moskovits M, Munden R, et al. (2003) Current rectification in a single $\mathrm{GaN}$ nanowire with a well-defined $\mathrm{p}-\mathrm{n}$ junction. Appl. Phys. Lett. 83, 1578-80.
[64] Kovtyukhova NI, Martin BR, Mbindyo JKN, Smith PA, Razavi B, et al. (2001) Layer-by-layer assembly of rectifying junctions in and on metal nanowires. J. Phys. Chem. B 105, 8762-69.

[65] Rotkin S. V., Ruda H. E., Shik A. (2003) Universal description of channel conductivity for nanotube and nanowire transistors. Appl. Phys. Lett. 83, 1623-25.

[66] Yan Voon L. C. L., Willatzen M. (2003) Electron states in modulated nanowires. J. Appl. Phys. 93, 9997-10000.

\section{Cite this article as :}

Kruti Wohra, Arun Kumar Diwakar, Anant G. Kulkarni, "Novel Properties of Semiconductor Nanowires", International Journal of Scientific Research in Science and Technology (IJSRST), Online ISSN : 2395-602X, Print ISSN : 2395-6011, Volume 8 Issue 5, pp. 345-354, September-October 2021. Available at

doi $\quad$ : https://doi.org/10.32628/IJSRST218552

Journal URL : https://ijsrst.com/IJSRST218552 